\title{
Space Mining Robot Prototype for NASA Robotic Mining Competition Utilizing Systems Engineering Principles
}

\author{
Tariq Tashtoush ${ }^{1 *}$, Jesus A. Vazquez ${ }^{2}$, Julian Herrera ${ }^{3}$, Liliana Hernandez ${ }^{4}$, Lisa Martinez ${ }^{5}$, Michael E. Gutierrez ${ }^{6}$,
} Osiris Escamilla ${ }^{7}$, Rosaura E. Martinez ${ }^{8}$, Alejandra Diaz ${ }^{9}$, Jorge Jimenez ${ }^{10}$, Jose Isaac Segura ${ }^{11}$, Marcus Martinez ${ }^{12}$

School of Engineering, Texas A\&M International University, Laredo, TX, 78041 USA

\begin{abstract}
The 2017 National Aeronautics \& Space Administration (NASA) Robotic Mining Competition (RMC) is an outstanding opportunity for engineering students to implement all the knowledge and experience that they gained in the undergraduate years, in building a robot that will provide an intellectual insight to NASA, to develop innovative robotic excavation concepts. For this competition, multiple universities from all over the U.S. will create teams of students and faculty members to design and build a mining robot that can traverse, mine, excavate at least $10 \mathrm{~kg}$ of regolith, then deposit it in a bin in the challenging simulated Martian terrain. Our team's goal is to improve on our current design and overcome DustyTRON 2.0's limitations by analyzing them and implementing new engineering solutions. The process to improve this system will enable our team members to learn mechanical, electrical, and software engineering. DustyTRON 3.0 is divided into three subteams, namely, Mechanical, Circuitry, Software sub-teams. The mechanical team focused on solving the mechanical structure, robot mobility, stability, and weight distribution. The circuitry team focused on the electrical components such as batteries, wiring, and motors. The Software team focused on programming the NVidia TK1, Arduino controller, and camera integration. This paper will outline the detailed work following systems engineering principles to complete this project, from research, to design process and robot building compete at the Kennedy Space Center. Only 54 teams were invited to participate from all over the US and DustyTRON team represented the state of Texas and placed the 29th and awarded the "Innovative Design" award.
\end{abstract}

Keywords-NASA robotic mining competition; mining robot; ice regolith; autonomous; NASA; space exploration; systems life-cycle, mechanical structure design, control system, systems engineering; software development

\section{INTRODUCTION}

As a leader in space exploration, the National Aeronautics and Space Administration (NASA) developed several unmanned robots, which were sent to the Moon and Mar in exploration missions to navigate the highly hazardous planets ecosystem and mine the available resources that will be converted to the needed energy (Oxygen and Hydrogen) before sending any human astronauts [1-12]. This technology provided the highest level of human safety and lowered space transportation costs.

The NASA Robotic Mining Competition (RMC) was started to engage university-level engineering students to de- sign, build, operate and compete with a robot that can be sent to space for a Martian chaotic terrain exploration. The off-world mining mission will be simulated where the robot will traverse and excavate simulated resources called regolith (Black Point-1 or BP-1) and ice (gravel), then return and deposit the excavated mass into a collector bin.

The eighth annual NASA Robotic Mining Competition (RMC) took place on May 22-26, 2017 at the Kennedy Space Center. This engineering challenge brought fifty-four U.S. university teams came to compete and show their unique and creative robotics design. DustyTRON Robotic team from Texas A\&M International University (TAMIU), fulfilled the competition goals based on NASA guidelines and RMC requirements [13-16]. This work marked our third participation in the RMC competition.

Each robot will have two ten-minutes trials to complete the mission. The field will be a $3.78 \mathrm{~m} \times 7.38 \mathrm{~m}$ arena which will be separated into three sections: starting area, obstacle area with rocks and craters, and a mining area. At the beginning of each trial, the robots were placed in the starting area at random positions and orientations. Then robots must traverse through the obstacle area which will contain two craters and three randomly placed rocks to reach the mining area. Once in the mining area, the robot needs to excavate then return to the starting area where a collection bin will be located to deposit the collected regolith. If time permits the robot will return to collect more regolith from the mining area.

The paper is organized as follows: Section II covers the available literature and NASA explore the space activities, Section III is a system requirements summary, Section IV illustrates all preliminary designs, Section V describes concept operation, Section VI shows the different systems' hierarchy, Section VII details the robot interface, Section VIII is risk management analysis, Section IX is the trade-off analysis, verification of System Meeting Requirements in Section X, Section XI reliability, Section XII summarizes the competition results, and Section XII is the paper conclusion and the future plan.

\section{LITERATURE REVIEW}

NASA's efforts and Robot exploration have always been around for many years where robots are used to collect data 
and to see how their actions and experiences can help us figure out ways to reach and live in space to enhance the future of mankind. Going back to the moon and exploring Mars have always been a goal for the U.S in the past centuries. Lunar mission and deep-space exploration can comply with the Global Exploration Road-map and the National Research Council. This mission name is called ALCIDES. ALCIDES will use some of the previous systems that were used in the HERCALES exploration, such as the Orion module, the Boeing Reusable Lander, the Ariane 6, the Falcon Heavy, the Space Exploration Vehicle, the Space Launch System, and the Evolvable Deep-Space Habitat placed in EML2. Robots and humans will need to work together to meet their goals, autonomously, and cooperate utilizing all the available technologies nowadays.

NASA Robotic Mining Competition (RMC) was stated due to recent NASA missions to Mars' discoveries, robots such as "Curiosity" and orbiting satellites taking pictures and videos showed a large amount of water in form of water ice and hydrated minerals on Mars [13-21]. Water sources formed millions of years ago were determined to be a result of clay and clay-like minerals on the surface or underground of Mars and Moon. Collecting these resources especially water will allow the humans' dream of living off the mainland. These resources can be utilized to provide humans with the required energy for rocket propellants, growing plants and sustaining astronauts, and protecting them in such a harsh environment. These minerals sources must be mined from the surface or buried deep in the ground.

NASA Robotic Mining Competition is a challenge for university-level undergraduate students from all over the United States (US). Students are tasked to design and construct a space-capable robot to traverse simulated Martian terrain and conduct a complete mining mission for the water and minerals. The mining robot must excavate the regolith simulant and/or the ice simulant that is located $30-50 \mathrm{~cm}$ deep then travel back to the simulated space station collection bin to deposit the collected resources. In addition to the fact that the robot must be space-focused, NASA added few complexities to the challenge such as the robot has to be limited in size and weight, can tolerate the abrasive characteristics of the regolith, can be teleoperated or completely autonomous, and power/bandwidthefficient.

Students participating in this competition can develop innovative robotic excavation concepts that allow NASA can use such excavation devices for future missions to advance human spaceflight and NASA space exploration operations. More info about this competition can be at https://www.nasa.gov/offices /education/centers/Kennedy/technology/nasarmc.html.

The NASA RMC started in its original format in 2010 as NASA Lunabotics Competition [14]. In 2011, it was open to undergraduate and graduate student teams enrolled in colleges or universities worldwide. But in 2014, due to NASA budgetary constraints, the competition was limited to teams from United States colleges or universities. In 2020, NASA transited to a Lunar-focused competition, and Table I] represents the competition year, name, and the allowed countries to participate [6].
TABLE I. NASA Robotic Mining COMPETITION History [6]

\begin{tabular}{|l|l|}
\hline Competition Year and Name & Competition Participants \\
\hline (2010) Lunabotics & USA \\
\hline (2011) Lunabotics & $\begin{array}{l}\text { USA, Bangladesh, Canada, Colombia, } \\
\text { India, Spain }\end{array}$ \\
\hline (2012) Lunabotics & $\begin{array}{l}\text { USA, Bangladesh, Canada, Colombia, } \\
\text { India, Mexico, Romania, South Korea }\end{array}$ \\
\hline (2013) Lunabotics & $\begin{array}{l}\text { USA, Australia, Bangladesh, Canada, } \\
\text { Colombia, India, Mexico, Poland }\end{array}$ \\
\hline (2014-2019) RMC & USA \\
\hline (2020-present) RMC: Lunabotics & USA \\
\hline
\end{tabular}

Many previously participating teams in NASA RMC presented their robots' design and operation following NASA requirements [16, 22, 28].

DustyTRON team utilizes this paper to present the implementation of system engineering concepts and processes in real-life problems and innovative solutions of space mining robots. The team participated previously in NASA RMC where they built mining robots DustyTRON 1.0 (2015) and 2.0 (2016), as shown in Fig. 1 and 2, respectively.

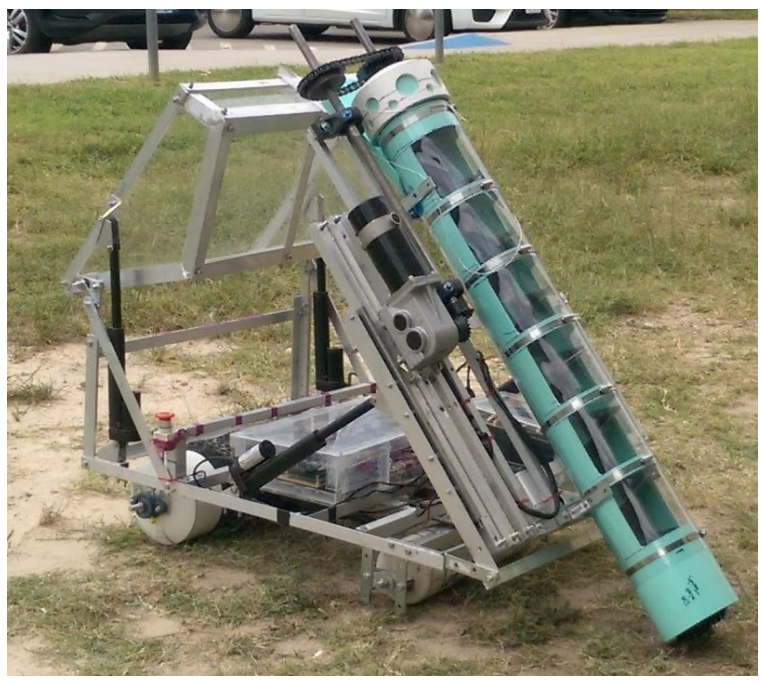

Fig. 1. DustyTRON 1.0 Robot - RMC 2015 [6].

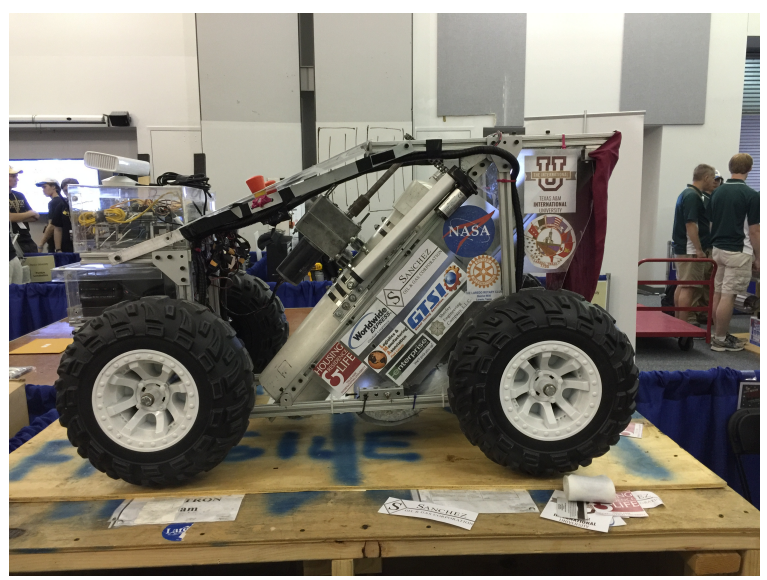

Fig. 2. DustyTRON 2.0 Robot - RMC 2016 [6].

The DustyTRON 3.0 is the improved design of 
DustyTRON 2.0, and the team consists of students that have participated before, seniors taking the class, and underclassmen interested in constructing a mining robot. DustyTRON 3.0 has a similar overall mechanical structure as DustyTRON 2.0, but with several improvements. Additionally, this paper includes a detailed analysis of the fully functional mining robot DustyTRON 3.0 to meet certain specifications including size dimension $(1.5 \mathrm{~m} X 0.75 \mathrm{~m} X 0.75 \mathrm{~m})$, weight $(80 \mathrm{Kg} \max )$, and mechanism (traverse, excavate, and deposit). The team's design theory and Quality Function Deployment (QFD) analysis will be the core of this project. Several designs were developed and evaluated based on multiple criteria such as design to build, mobility, weight, and budget, then followed by decisionmaking to select one optimum design.

DustyTRON 3.0 was split into three sub-teams: 1) mechanical design and construction, 2) electrical circuitry design, and 3) software development.

- Mechanical design and construction team focused on a robot structure development where the robot must have a strong structure that moves easily while keeping lightweight, an excavation mechanism, and a regolith collection and deposit mechanism. They will improve the rigidity of the middle structure of the robot, where the excavation mechanism will be mounted and enhance the steering system.

- Circuitry team will link the mechanical and software components together to achieve a fully functional robot. They will improve on the electrical components and storage for easy accessibility and monitor-ability, and safe from any external influence. Cables will be routed in different layouts so that troubleshooting and repairs will be easier and faster in case of a problem, which will reduce the risk of an electrical short, electrical interference, or electrical failure significantly.

- Software development team worked on developing the autonomous functionality by moving to SystemOn-Chip (SoC) and microprocessor system. Intercommunication between the SoC and Microprocessor will be conducted through a serial interface while a secure connection between the robot and the control station will be used. The autonomous mode will utilize OpenCV (Computer Vision) library for image and object detection for Xbox One Kinect and IP cameras.

DustyTRON 3.0 team planned to build a robot based on DustyTRON 2.0 in order to reduce the total budget, by providing improvements and solutions to last year's design problem. The mechanical team's improvements will include motors and steering systems modifications, which was estimated to be $\$ 2000$. For both circuitry and software teams, the budget estimation was $\$ 1000$ because last year components will be recycled and used for this year's robot. Table [II shows the estimated budget and the actual cost.

The actual budget of DustyTRON 3.0 had been changed along the building process due to sudden failure of electrical components such as motor drives and voltage regulators, hardware parts, such as t-slotted beams deformation, and wheel design changes. With extensive research and some educational discounts and donations, the total cost was lowered. One
TABLE II. DUSTYTRON 3.0 Estimated AND ACTUAL Budget

\begin{tabular}{|l|l|l|}
\hline Team & Estimated Budget & Actual Budget \\
\hline Mechanical & $\$ 2000$ & $\$ 640.67$ \\
\hline Circuitry & $\$ 500$ & $\$ 1100.72$ \\
\hline Software & $\$ 500$ & $\$ 0.00$ \\
\hline Total & $\$ 3000$ & $\$ 1741.39$ \\
\hline
\end{tabular}

important note is that the software team did not have to make any significant purchases to prepare for the 2017 NASA RMC competition, as previous years of competing had provided the team with all the physical components to build the software needs. The digital aspect of needed materials required no purchasing since programs like TurboVNC, PuTTY, Arduino IDE, and Ubuntu were all free.

\section{SySTEM REQUIREMENTS}

The Project aimed to develop an inexpensive multi-purpose space exploration rover system that is capable of image capturing, rock mining, and data collection. Many researchers and engineering teams [29.41] worked on developed new exploration technologies for Moon and Mars applications.

This design effort started by gathering and deriving the requirements from NASA RMC competition rules and regulations as a benchmark. These requirements were followed and frequently checked to meet the competition regulations and goals. As system engineers, the team split the project into functional subsystems and identify their interaction as they are the base of the generated concepts and allowed to create a scoring rubric with respect to meeting the requirements. The main requirements are listed in Table III

TABLE III. SYSTEM REQUIREMENTS EXTRACTED FROM [23-25]

\begin{tabular}{|l|l|l|}
\hline $\begin{array}{l}\text { Requirement } \\
\text { Type }\end{array}$ & Action & Specifications \\
\hline $\begin{array}{l}\text { Performance } \\
\text { Requirements }\end{array}$ & $\begin{array}{l}\text { Excavate } \\
\text { Regolith }\end{array}$ & $\begin{array}{l}\text { Excavate an adequate depth to reach the ice } \\
\text { simulant }\end{array}$ \\
\cline { 2 - 3 } & $\begin{array}{l}\text { Collect } \\
\text { Regolith }\end{array}$ & Storage to collect the excavated regolith \\
\cline { 2 - 3 } & $\begin{array}{l}\text { Deposit } \\
\text { Regolith }\end{array}$ & $\begin{array}{l}\text { Deposit the collected regolith onto a bin } \\
\text { located at end of the simulated terrain }\end{array}$ \\
\hline $\begin{array}{l}\text { Design } \\
\text { Requirements }\end{array}$ & Dimensions & $\begin{array}{l}\text { Maximum measurements of } 1.5 \mathrm{~m} \text { in length, } \\
\text { and } 0.75 \mathrm{~m} \text { in both height and width }\end{array}$ \\
\cline { 2 - 3 } & Weight & Maximum weigh of $80 \mathrm{Kg}$ \\
\hline
\end{tabular}

\section{DUSTYTRON 3.0 PRELIMINARY DESIGNS AND IMPROVEMENTS}

The main goals of any systems engineer are continuous improvement and performance enhancement; therefore, the team started by analyzing DustyTRON 2.0 robot and evaluating its performance. DustyTRON 2.0 had some challenges in the area of electrical motors used for wheel and mobility and steering limitations. Hence, DustyTRON 3.0 mechanical team invested significant time to redesign the wheels and steering system to develop various alternatives such as changing the wheel design, acquiring stronger motors with high torque to handle the robot weight, and implementing a new steering mechanism. Additionally, the software team tackled the current code by providing a cleaner and more functional code for both Arduino and controllers, while the circuitry team focused on enhancing the electrical circuit, motor drivers, cable management, wiring harnesses, layout, and power management. 


\section{A. Design Development}

1) Design 1: This design utilizes an auger to excavate the simulated Martian regolith and a Plexiglas box as storage, as shown in Fig. 3. The frame will be built using T-slotted beams, and PVC pipe, within the following dimensions $1.4 \mathrm{~m}$ (length) and $0.75 \mathrm{~m}$ (width and height).

The auger system has an auger $(0.513 \mathrm{~m}$ length, and 0.152 $\mathrm{m}$ diameter) and will be enclosed using a PVC pipe $(0.152$ mm ID). The auger will be attached to a fabricated V-shaped bracket that will be rotated on a pivot point, using two 24-volt heavy-duty servo motors. The home position of the auger is laying inside the collection box. Plexiglas box is sized and positioned to allow the auger to dump and store the regolith. This box will be dumped using 12" linear actuators. Plexiglas was used for the collection box because of its durability and lightweight and has been proven to be able to contain the regolith and protect other components quite efficiently. Electrical boxes will be mounted to the side of the collection box. All four wheels will be 16-inch diameter and 4-inch-wide powered with high torque motors.

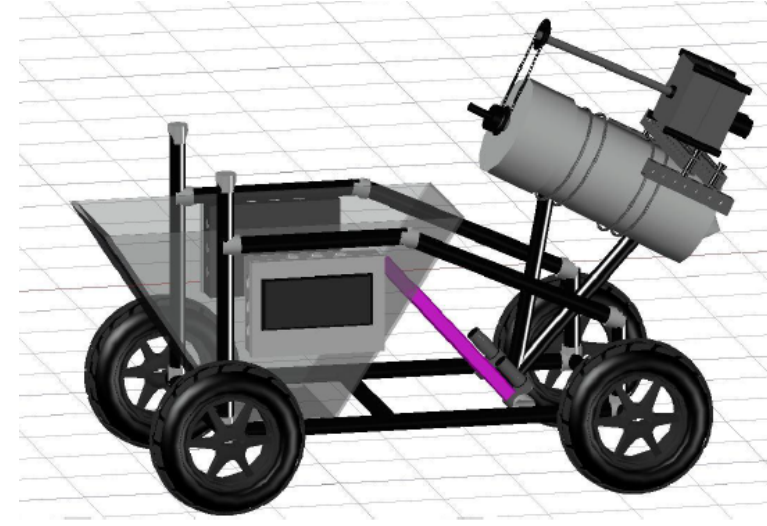

Fig. 3. DustyTRON 3 Mechanical Structure Design 1.

2) Design 2: The structure design 2 close to the DustyTRON 2.0 with few necessary changes as shown in Fig. 4. The team decided to locate the electrical boxes to the sides of the structure while keeping the auger angle fixed and increasing the collection box by modifying the conveyor belt system. The conveyor belt system changed to follow an Lshape, which increases the collection box size while being able to move the regolith from the bottom to the dumping point behind the robot.

3) Design 3: As shown in Fig. 5, the robot design had been developed to include significant modifications such as the dumping system which consists of a single inclined conveyor belt but longer so it can go beyond the rear wheels. Each set of two wheels (front and back wheels) will be attached to a perforated steel tube to create the steering system, this will be attached to the middle frame using two linear bearings and two linear actuators allowing to adjust the height of the robot when needed. In addition, the previous bulky wheels had been eliminated in favor of lighter thinner wheels that would perform the same job.

4) Final design: Various major changes had been conducted to improve the team's design as can be noticed in

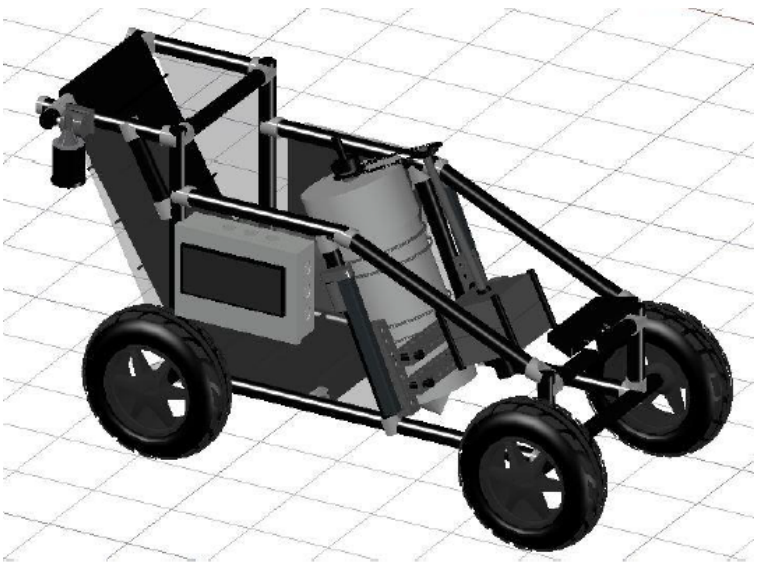

Fig. 4. DustyTRON 3 Mechanical Structure Design 2.

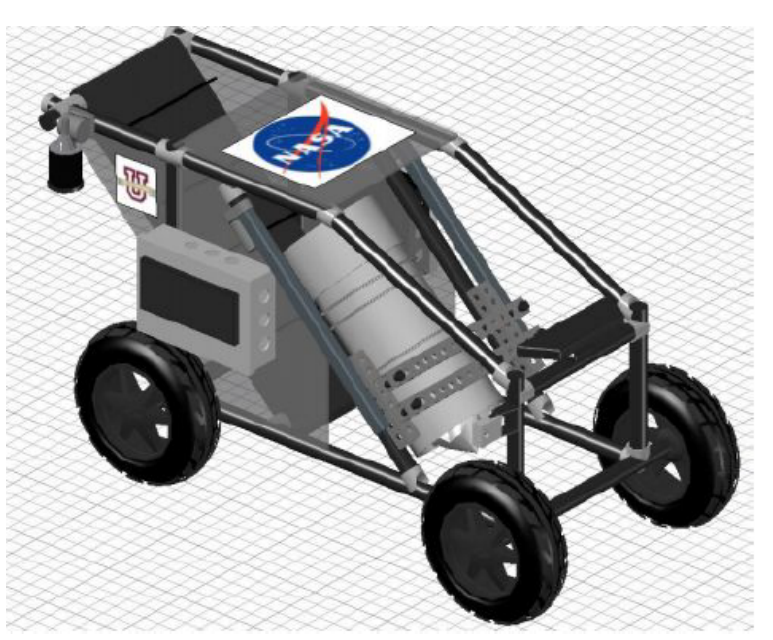

Fig. 5. DustyTRON 3 Mechanical Structure Design 3.

Fig. 6. The first and most important change is the auger system, which became independent of the middle structure as its tilting angle can be changed using two linear actuators while it can slide down using another linear actuator. Also, wheel-motor attachment has been designed and 3D printed in TAMIU facilities. The team began to build this robot for many reasons; the most important one is the weight, and structural rigidity, and stability which was achieved by using the lightweight T-slotted 80/20 bars. Additionally, the sliding mechanism allowed the auger and wheels to move easily without affecting the frame integrity.

DustyTRON 3.0 requires four (4) independent wheels, ten (10) linear actuators, and one (1) 6-inch inner-diameter excavating auger, which was the foundation of the circuit design. To be able to power all these components with sufficient power distribution and move the $80 \mathrm{Kg}$ robot, the team decided to use the following: four 24-Volt motors for the wheels, two 24-Volt motors for the auger, which was confirmed at the testing stage, where power was enough to rotate the auger at the desired speed and using LiPo battery was the best power source for the system.

With the new DustyTRON 3.0 design, a new software configuration and code had been developed to allow simpler 


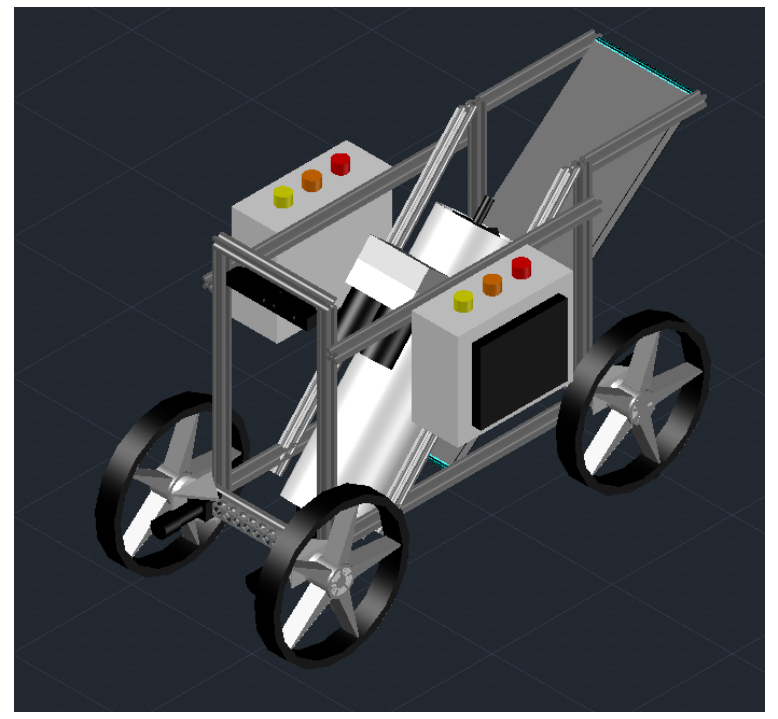

Fig. 6. DustyTRON 3 Mechanical Structure Final Design.

operation and control of the robot. The software team started by reforming the Arduino codes to be easier to read and userfriendly, which allowed easy and precise executable commands utilizing Arduino open-source libraries for the robot individual components, such as the servo library to control Axis 206 Network Camera. While the Pololu motor driver will be using the previously developed library to control all motors and linear actuators.

The changes in the design of DustyTRON 3.0 are now profoundly different, ranging from the wheels to the angle of the auger. The team pushed that boundary of what can be done and showed how much System Engineers can improve on already proven designs.

\section{B. DustyTRON 3.0 Improvements}

1) Mechanical team improvements: The main focus for the mechanical team is to fix the steering system of the DustyTRON 2.0 robot, which consists of the wheels, wheel attachment, and motors. While aiming to solve that issue, the team wanted to keep the four-wheel drive (4WD) options as it helps the robot to overcome any obstacle such as a rock or a crater. After extensive research, the team had to find a solution or a method to attach the motor to the middle bar which will act as the rack/structure of the steering system. This bar is a hollowed steel bar that will allow the team to have a strong structure while keeping it lightweight. The original idea required the use of two-wheel casters per side to make a pivotal point and the motor will be mounted in between both wheel casters. The four rods attached to the wheel caster shown in Fig. 7 will be used to securely attach the motor in place.

After detailed analysis, this idea had been developed to include a square perforated steel tube, this new design will use two-wheel casters only and the tube will be used to mount the linear actuator brackets. Fig. 8 shows the steering system with the perforated steel tube and linear actuators. With one caster at each end, the motor can be attached directly to the inner vertical wall of the caster, which means less mechanical interference and easier rotational motion for steering. The shaft

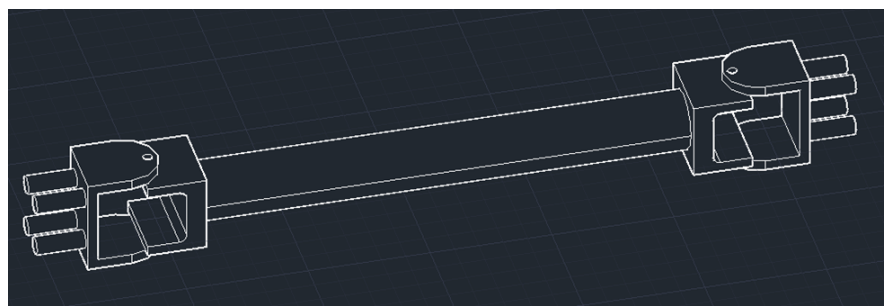

Fig. 7. DustyTRON 3 Proposed Steering System.

would be the only thing coming out of the caster which connects both wheels and will be connected to the robot structure.

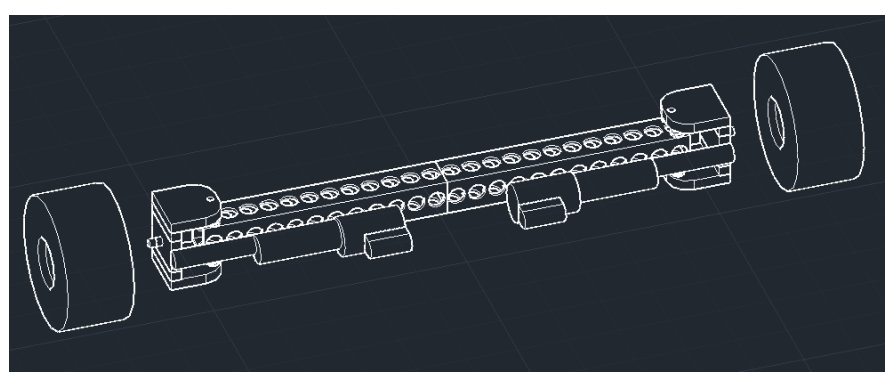

Fig. 8. DustyTRON 3 Final Steering System.

After finalizing the motor attachment, the team found a solution to attach the motor shaft to the wheel by building a two-parts wheel hub. This new hub consisted of an Alumni $8 \mathrm{~mm}$ screw hub which will be attached directly to the motor shaft. While the second part will be a special part that will match with the grooves of the wheel and both parts will be attached together using bolts and locknuts. Unfortunately, the team did not have access to the Computer Numerical Control (CNC) machines to manufacture the complete wheel hubs; therefore, the team decided to utilize the 3D printer to create the hub-wheel attachments. These hubs went through various phases until a perfect fit was found, Fig. 9 shows the different phases/designs of the wheel hubs.

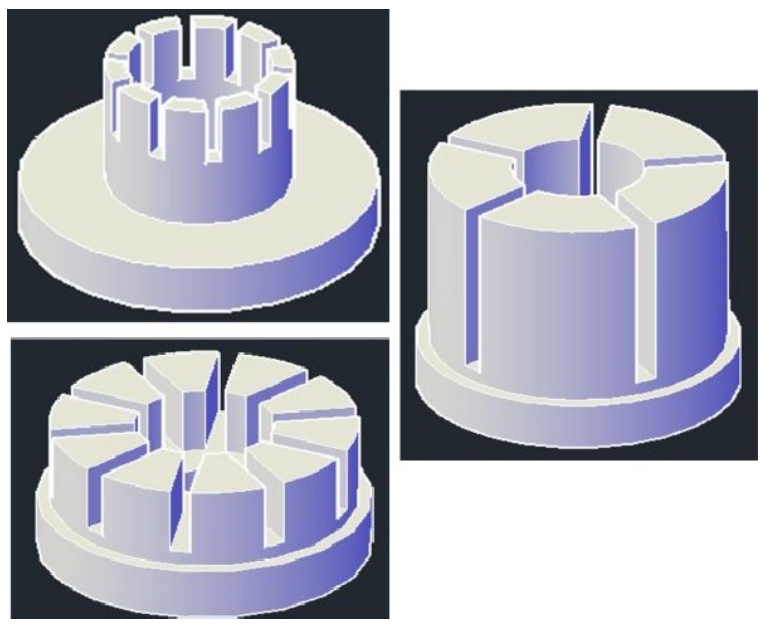

Fig. 9. DustyTRON 3 Wheel Hubs.

DustyTRON 3.0 steering system will be very efficient and 
have easier maneuverability compared to the previous robot steering, which will reduce the time required for steering in the competition runs. In addition, springs and wheel bearing had been added to the caster in order to add more flexibility and enhance the rotation movement of the caster as shown in Fig. 10

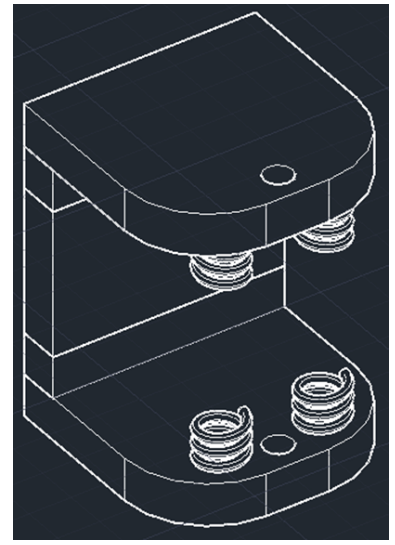

Fig. 10. DustyTRON 3 Caster Design.

The selected wheels for the final design, were 14inches in diameter and 1.75 inches in width. This will help the team to have a smaller footprint while steering and will allow the team to decrease the robot turning angle.

Another significant improvement is that DustyTRON 3.0 will contain a suspension system that will control the height of the robot using linear actuators, which will change the clearance between the auger tip and the ground from 4 inches to 10inches. This height difference would allow the robot to go over small to medium-sized obstacles. Several designs were taken into consideration before selecting the final design. One more improvement to the mechanical structure is providing more rigidity to the auger structure; this was achieved by adding two t-slotted bars to join the front and back ends of the robot. Two linear actuators had been added in order to change the auger tilting angle and reduce the vibration transmission to the robot structure.

2) Circuitry team improvements: The circuitry team focused on solving the electrical components layout and wiring issues. The team decided to purchase higher quality electrical boxes, design a better component layout within the component boxes, better component box placement onto the robot and decided to install fans within the motor control box. Having that in mind, the team started working on designing the layout within the component boxes to organize the cables to harness the wires in a way to reduce the cable length. This reduces the risk of having an excess of loose cables and reduces the electrical noise that might affect the microcontroller and motor driver's performance. These component boxes will house and protect all the electrical components that are required for the robot operation, which resulted in two-component boxes that will be explained below.

Electrical Box 1: Main Brain Box The first electrical box is labeled Main Brainbox since is considered the main computer of the robot. It consists of a $14.8 \mathrm{~V}$ Lithium Polymer RC
Battery, power on/off switch, power analyzer, fuse, 1 E-Stop button, voltage step down, NVidia TK1 will be connected to LAN line, USB HUB, and display with HDMI cable. The NVidia TK1 will be powered through a step-down voltage regulator as it requires 12 volts. The USB HUB will be plugged in the NVidia USB 3.0 port to power the Arduino, a rearview camera, keyboard and mouse, and a data terminal for the Xbox Kinect camera. Fig. 11 shows the battery, power switch, power analyzer, E-Stop, fuse, step down, and NVidia connection in this box.

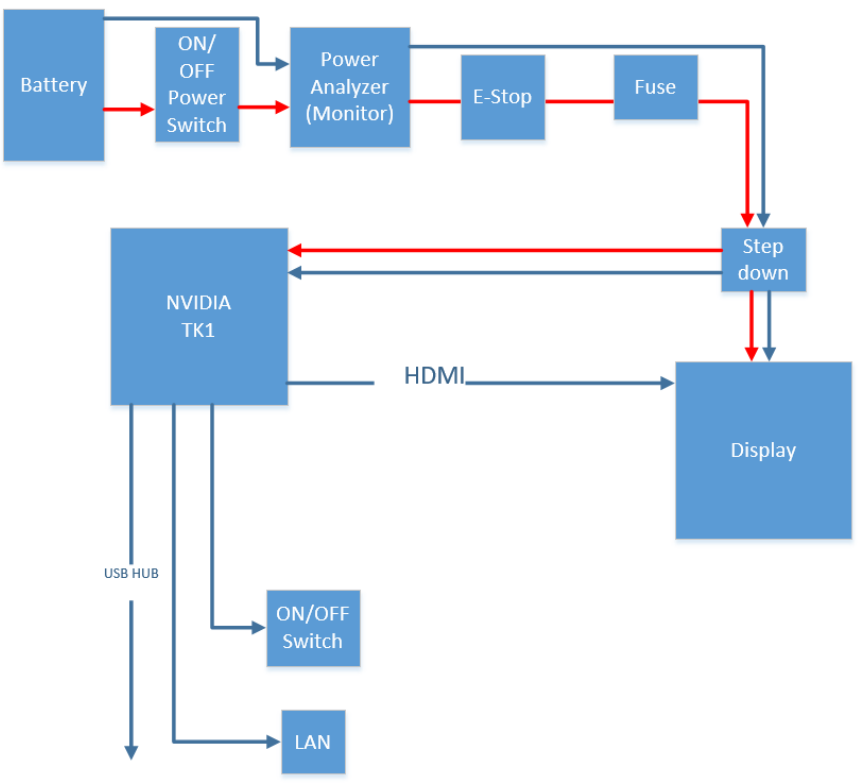

Fig. 11. DustyTRON 3 Robot - Main Brain Box: Component Circuit Diagram.

Electrical Box 2: Motor Control Box Electrical box number 2, labeled as Motor Control Box, is where all the other electrical components are connected to all hardware components that are vital for the robot's physical movement. This box contains 1 power switch, 1 power analyzer, 1 emergency stop button, 1 battery, 1 eight fused-output power distributor board, 3 fans, USB HUB, 1 operation flashing light, 1 Arduino, and 6 motor drivers. The $22.2 \mathrm{~V}$ battery is connected in series to the emergency stop button, which is then connected to the power analyzer that is connected to the power switch. The emergency stop button and the fuses in the power distributor work as a method of safety to protect the circuit from any malfunctions. The operation flashing light is connected directly to the power distributor and it will be used as an indication of robot reediness. The power distributor contains an on and off switch and eight fused power outlets. The terminals to the motor drives are connected to six of the power distributor outlets. One of these fused power outlets will be connected to a step-down voltage regulator that had been adjusted to output 12 volts only to power the cooling fans, and a second stepdown voltage regulator will be also adjusted to 12 -volt output to power the Kinect.

The purpose of the fans and the heat sinks attached to the motor drivers is to extract the heat created within the motor control component box. Two of the six motor drivers will be connected 
to each motor on the wheels, one motor driver for the front wheels and one motor driver for the back wheels. These motor drivers have dual channel connections. The other motor drivers will be used for the steering and suspension actuators, and the slider actuators and conveyor belt. These Pololu motor drivers are dual channels that are capable of delivering up to $12 \mathrm{~A}$ each. Therefore, by combining both channels into a single, that will have provided the motor attached with current up to 24A. The auger and horizontal auger actuators are connected to be in single-channel mode. Fig. 12 illustrates the components and the connection in the motor control box.

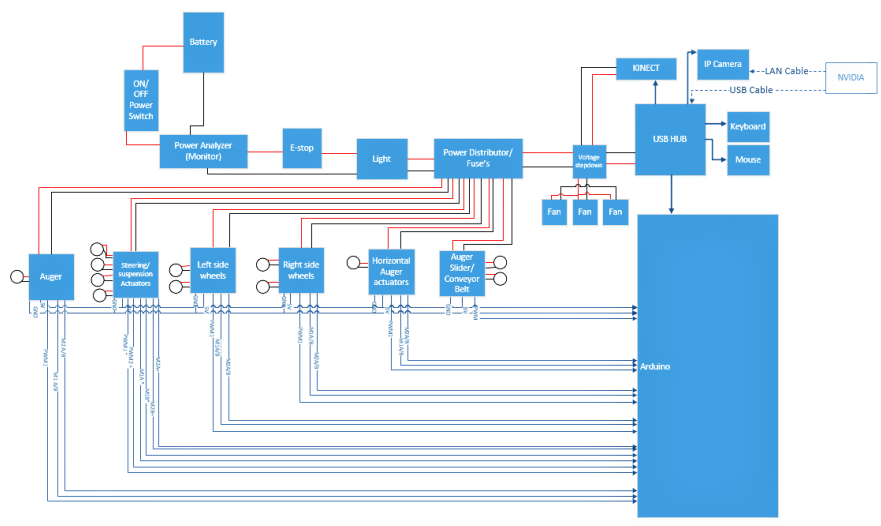

Fig. 12. DustyTRON 3 Robot - Motor Control Box: Component Circuit Diagram.

These electrical boxes are made of thick plastic in comparison to the previous robot's boxes that were made out of plexiglass. These boxes provided the team with a better structure to mount the E-stop and power switches without influencing the integrity and weather resistance of the boxes. These boxes will be mounted in the available space between the wheels and on the sides of the robot, with removable mounting brackets. This location will provide better weight distribution and having the electrical boxed in an elevated position, which will keep the components safe far from highly active moving components and allow easier access for maintenance, troubleshooting, and even parts replacement when needed.

Another improvement was the addition of a fused power distributor board that can handle the supplied voltage and current; since this will regulate the voltage into the motor drives, operation flashing light, E-stop, power switch, and voltage step down. Lastly, the circuitry and software team decided to install a 10-inch display monitor, that can be attached to the main electrical box that houses the NVidia, which will make troubleshooting easier.

3) Software team improvements: The software team focused their effort on creating an optimized code that will be used to control all the linear actuators and motors within the robot. In addition, the software team worked on enhancing the code for the manual control mode and provided a cleaner, simplified, and user-friendly code that will be used to interface the Xbox controller to the NVidia microprocessor. Optical sensors within Xbox Kinect and a servo camera were added to the robot for autonomous operation and obstacle detection and avoidance. The Kinect camera was placed at the font to frontal environment scan, while the servo camera is was mounted in the back of the robot to monitor and regulate the deposit mechanism. DustyTRON 3.0 used the Jetson TK1 Graphics Processing Unit (GPU) to facilitate autonomous operation by implementing wireless communication and computer vision.

\section{CONCEPT OPERATIONS}

DustyTRON 3.0 will have a better steering system, new wheels, and a new overall structure design. The weight will decrease due to battery change and the frame will still be constructed by $80 / 20$ T-Slotted bars because of the lightweight and easy manipulation. To excavate the simulated Martian terrain, DustyTRON 3.0 will use a double helix auger that is powered with dual motors with a gearbox of $47: 1$ ratio and two linear actuators to move the auger into the ground. DustyTRON 3.0 has 14inches $X 1$.75inch wheels in order to have better steering and powered with $24 \mathrm{~V}$ high torque motors with a $295: 1$ great box ratio. The suspension mounted on the structure of the robot is meant to lift the robot, in order to go over the larger rocks on top of the first layer of BP1 and to lower the robot once the digging process starts. DustyTRON 3.0 was redesigned to operate autonomously with the use of two cameras, a microprocessor, and a graphical processing unit. The designed autonomous mode utilizes a Microsoft Xbox 360 Kinect camera with an IR sensor that provides video data to the CPU, and the NVidia TK1, for object detection. The second rear servo IP camera provides the team video data that regards to regolith deposit.

In case of autonomous mode failure, DustyTRON 3.0 can be controlled over WiFi by two Xbox 360 controllers from a max distance of about 50 feet. This manual control of the robot is established by sending simple 8-bit commands that resemble keyboard strokes, to an Arduino Mega 2560 unit that is directly connected to an NVidia TK1. Whether the robot is in manual or autonomous mode, NVidia TK1 will pass the commands to the Arduino that directly controls all motor drivers and operate the wheel motors and actuators.

\section{SYSTEM HIERARCHY}

The relationship within the main components of each subteam can be illustrated using a system hierarchy diagram. For the mechanical team, the moving, excavating, and deposit system relationship is shown in Fig. 13. Circuitry and software sub-teams share several common components such as controller, NVIDIA GPU, Arduino, and motors. Both subteams will be working to assure both manual and autonomous operation of the robot. Manual control will be based on the pictures and live feeds provided by Xbox Kinect, the human operator will use an Xbox 360 controller to send the command through the controller computer to the robot's NVIDIA TK1 processor, which are interconnected through wireless WiFi and use SSH (Secure shell). This SSH was selected as it is an encrypted network protocol, and will prevent any unauthorized access to the robot's TK1.

In the autonomous mode, the Xbox One Kinect feed will be processed directly within the TK1 for object detection purposes, while the rear camera feed will be used for the regolith collection and deposit process. In both scenarios; manual or autonomous mode, TK1 will command the Arduino Mega, which is directly controlling the motor drivers and 
mechanical components. Fig. 14 shows the circuit hierarchy and Fig. 15 shows the software system hierarchy.

Additionally, the software team implemented a VNC communication to reduce the used bandwidth by compressing the video feed before broadcasting to the main control station. Within the robot system, serial communication and powering the Arduino was done using the USB port, which simplified the Arduino power circuit.

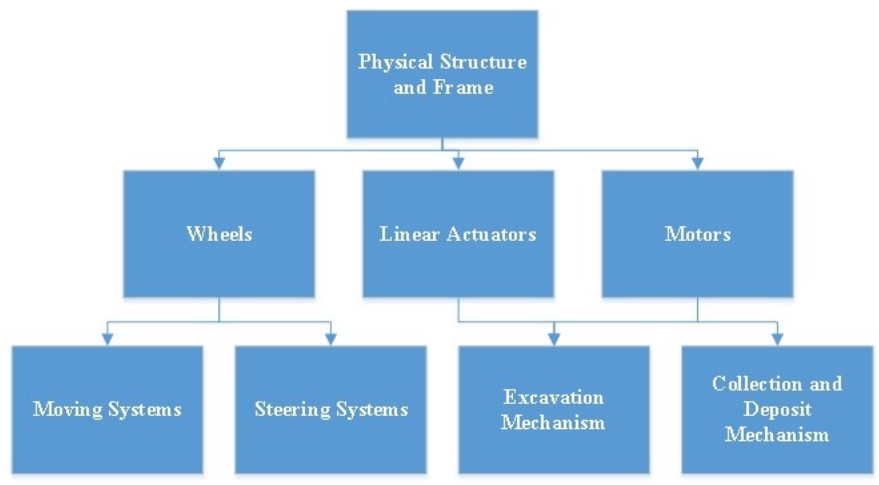

Fig. 13. Mechanical System Hierarchy for DustyTRON 3.0.

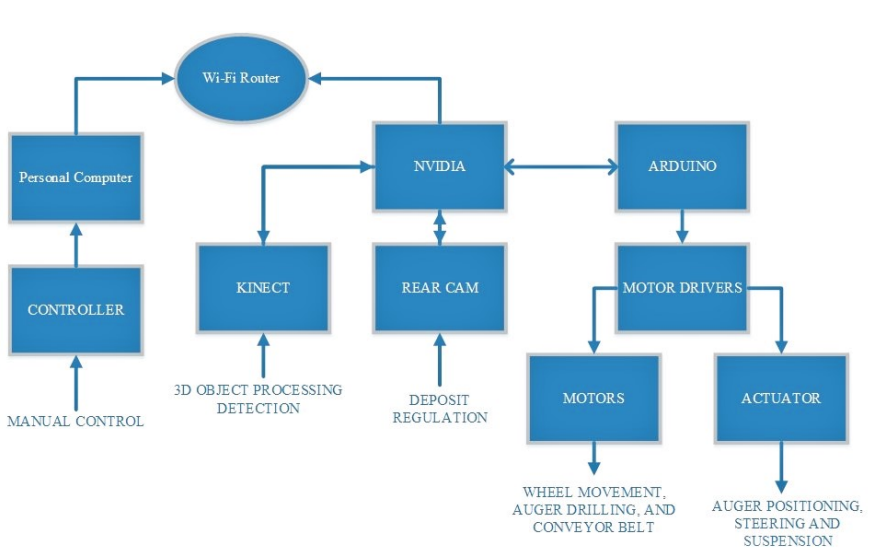

Fig. 14. Circuitry System Hierarchy for DustyTRON 3.0.

\section{ROBOT INTERFACE}

One of the main objectives of the DustyTRON 3.0 engineering design process was to create a reliable and maintainable interface between our subsystems. Fig. 16 shows a level diagram for components' interface for the mechanical, electrical, and software systems.

The robot interface was built using Ubuntu 14.04 as the NVidia TK1 operating system (OS) with different software such as Arduino Software IDE to communicate the Arduino Mega, Microsoft XNA to program the Wired Xbox 360 controllers that will be used for the robot manual control mode by developing a Visual Basic (VB) Code, while PuTTY was used for serial port communication between the NVidia TK1 and the Arduino to emulate Arduino's serial console to receive input data, and TurboVNC were used to establish two-way secure remote communication [42].

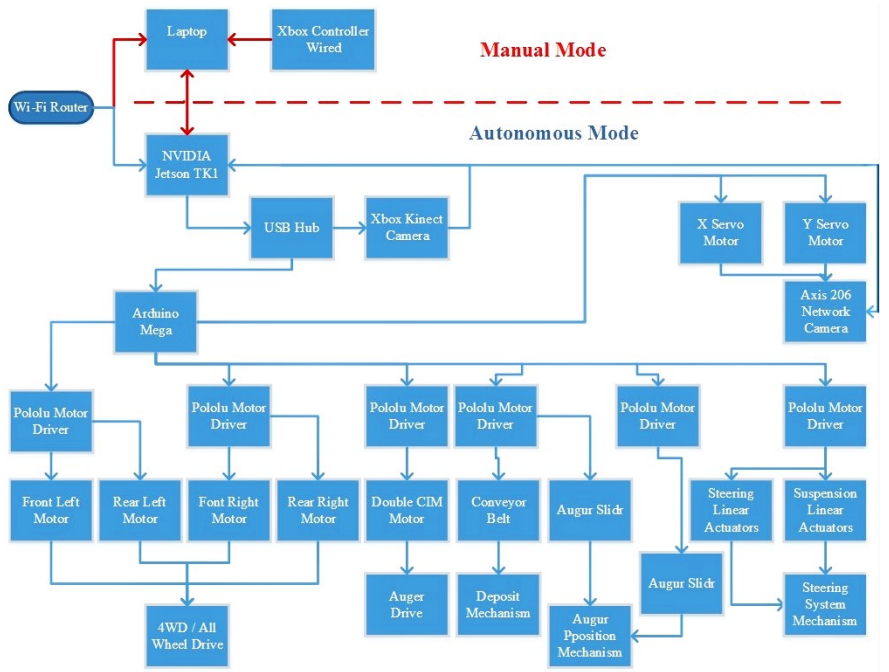

Fig. 15. Software System Hierarchy for DustyTRON 3.0.

Sub-Team Interfaces

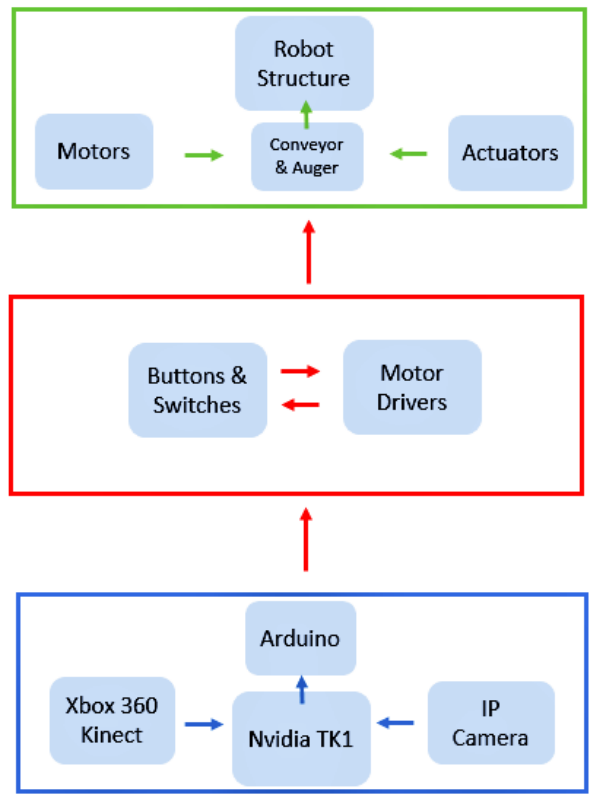

Fig. 16. Systems Interface for DustyTRON 3.0.

\section{RISK MANAGEMENT}

As a Systems Engineer, prediction and planning for the future is an essential task in any project. This step can be achieved by analyzing the system of interest for any possible failures and developing a ranking system for failure consequences on the overall performance of the robot and likelihood of happening, which will allow the team to adapt and prepare with a solution if issues arise. Each sub-team developed their risk matrices and they are as follow:

\section{A. Mechanical Team}

The Mechanical Team analyzed the mechanical structure and components and found the following risks that might occur 
during or before the competition:

- Failure to move: if the steering system cannot function, or fails to traverse to excavate. Major Consequences and Unlikelihood of Occurrence

- $\quad$ Failure to excavate: if the excavation (Auger) system is not working as expected due to a failed motor or chain or obstacles exist within the system. Catastrophic Consequences and Rare Likelihood of occurrence

- Failure to collect regolith: in the event where the collecting mechanism fails to hold regolith or the excavation mechanism is not providing enough regolith. Major Consequences and Moderate Likelihood of Occurrence

\section{B. Electrical Circuitry Team}

The risks that might occur during or before the competition were found to be:

- $\quad$ Failure of Circuitry: if the circuitry/cables fail and burn due to an unexpected overheat, which might lead to the entire component box failing. Major Consequences and Rare Likelihood of Occurrence

- Failure of Battery: if batteries failed to hold an electrical charge or not able to provide the required electrical power. Moderate Consequences and Moderate Likelihood of Occurrence

- $\quad$ Failure of Motor Drivers: if motor drive overheats or stops responding to the Pulse Width Modulation (PWM) signal. Major Consequences and Unlikelihood of Occurrence

- Failure of Motors: if the motor malfunctions and not able to rotate the attached mechanical component. Catastrophic Consequences and Unlikelihood of Occurrence

In the building phase, the circuit team was actively testing and verifying the proper operation of every single component to prevent any future issues. They simplified the circuit design to allow fast and easy components' diagnostic and replacement.

\section{Software Team}

For the software architecture design, some failures can be due to connection with a mechanical-related failure. The major risks that had been considered are as follows:

- Failure of feedback: if the connection fails to send feedback on possible problems or updates, or if the Kinect camera or IP camera loses signal, the team will be prevented from viewing the terrain. Minor Consequences and Unlikelihood of Occurrence

- Failure of NVIDIA TK1 power regulator: if the power circuit fails to provide the TK1 with the required 11.6-12.6 Volt, then the GPU will fail and go into limp mode. Moderate Consequences and Unlikelihood of Occurrence
- Failure of VNC connection: If the remote access connection fails to be established, then the robot won't be controlled manually or it might not be able to receive the autonomous start signal. Catastrophic Consequences and Moderate Unlikelihood of Occurrence

- $\quad$ Failure of programming OpenCV: if the vision and image analysis system fails to start then the robot won't run autonomously. Major Consequences and Rare Likelihood of Occurrence

- Failure to send a command to Arduino: if serial communication fails between the TK1 and Arduino, then the robot will fail to do the required mission as no movement will be executed. Catastrophic Consequences and Rare Likelihood of Occurrence

\section{TRADE-OFF ASSESSMENTS}

Using Quality Functional Deployment (QFD) method, the team was able to find their design's strength and worked on enhancing them using a trade-off assessment for every subteam.

\section{A. Mechanical Team Trade-off Assessment}

The robot's mechanical structure strength was found to be the independent controlled suspension, which will allow the robot to adjust its height to go over obstacles and rough terrain while adding extra components and weight to the robot.

\section{B. Circuitry Team Trade-off Assessment}

The previous design of the robot was based on utilizing six (6) $12 \mathrm{~V}$ 7Ah sealed batteries, which were $4.5 \mathrm{lb}$ each, the team decided to switch to two LiPo batteries (14.8V and 24V) which are lighter (2.6 lbs in total) but extremely powerful and careful circuit design is required to prevent any damage to electrical components such as TK1 or motor drivers. The circuit was improved by changing wires to thicker gauge (12 AWG), adding heavy-duty power distribution with fused ports, including fans to extract the heat within the electrical boxes, and batteries were protected using Fireproof Safe Bag. In addition, the VNH5019 Pololu motor drives were used although they require soldering and extra configuration for mono or dual channel. They provided superior performance and accuracy to a single motor or double motors control.

\section{Software Team Trade-off Assessment}

For manual control implementation, two wired Xbox 360 controllers were used to eliminate the wireless connection lag and it will allow the simplification and splitting of robot controlling tasks by having one person control the excavation system and another operate the robot mobility. The team chose to implement autonomy by using an Xbox Kinect Camera. If autonomy fails, the team will change to manual control to regain control of the robot. The Xbox Kinect camera was used instead of the PS3 Move camera. Although the PS3 Move camera used less power consumption and had a better resolution, the Xbox Kinect had integrated sensors that will allow the 3D mapping feature needed for the autonomous mode [43]. These integrated sensors balanced the power consumption 
consequence since having to get extra individual components would result in a similar outcome. Arduino Mega 2560 [44] was selected to control and command the motor drive utilizing its superior and stable PWM compared to the Jetson TK1. Axis 206 Camera was used for excavation and collection operation monitoring as it has two servo motors to control its aim in $\mathrm{X}$ and $\mathrm{Y}$ directions.

\section{REQUIREMENTS VERIFICATION}

To assure that the robot was designed and built to meet NASA regulations, the following requirements were checked frequently:

\section{A. Functional Requirements}

- Robot must traverse the simulated Martian terrain and excavate the needed regolith from the mining area.

- Tele- or autonomous operation of the robot.

- Sufficient size collection system to stored Regolith until the deposition.

- Obstacle avoidance in the arena.

- The robot's suspension shall be able to lift the rear or front end as desired.

- $\quad$ Robot code must be simple and easy to execute.

\section{B. Performance Requirements}

- The robot shall be able to start the mission from any assigned location or orientation.

- Collect and deposit $10 \mathrm{~kg}$ of BP-1 within the allowed 10-minute mission.

- Excavate BP-1 from the designated area only.

- Dust prevention and electrical components protection.

- $\quad$ Limited bandwidth and power consumption.

\section{Physical Requirements}

- Maximum weigh of $80 \mathrm{~kg}$.

- $\quad$ Self-sustained power with consumption monitoring and recording system.

- Initial dimensions of $1.5 \mathrm{~m}$ Length, $0.75 \mathrm{~m}$ width, and $0.75 \mathrm{~m}$ height.

\section{Safety Requirements}

- An emergency stop red button with a diameter of $40 \mathrm{~mm}$ in an easy and safe accessible position.

- All wire harnesses are securely attached and protected.

- Easy and secure connection to the robot control systems.

To assure that the robot is meeting all NASA RMC's requirements, the team verified their design in the testing phase by inspecting the Commercial off-the-shelf (COTS) parts used to perform as intended. Some of the inspected items are shown below:
- The extruded T-slot bars' integrity was inspected under loading and vibration conditions.

- Operate the twin spiral auger in a simulated sand field to assist its performance and measure the collected sand weight.

- Linear actuators and motors were tested before and after fitting it to the frame to make sure of their ability to move the robot.

- The conveyor belt system was tested to check its operation and ability to move the regolith from the collection box to the dumping location.

- Batteries were charged and monitored to guarantee they can last for the 15-20 minutes mission.

- Emergency-stop buttons were tested where the power to the whole robot was shut down safely.

- Axis 206 and Xbox Kinect camera functionally were tested.

\section{ReliabiLity}

To ensure the robot's maximum reliability of the robot, a few actions had been taken:

- Hardware team strengthening the structure and reduced the weight, improved the wheels, steering, and suspension systems to overcome the harsh terrain.

- Circuitry team arranged the electrical components to reduce and manage the cables and connections effectively, and by using LiPo batteries their contributed to reducing the robot's total weight.

- Software team utilized the serial communication and VNC connection secure the interface between NVIDIA, Arduino, and main control computer. Additionally, codes were updated and improved and Fig. 17 shows the updated pseudocode for the Arduino.

\section{COMPETITION Results Summary}

The DustyTRON robot shown in Fig. 18 was delivered to Kennedy Space Center in Florida to participate and compete against 53 robots from all over United State. The robot passed all the inspection procedures and after the competition runs, it placed the 29th and was awarded the "Innovative Design" for its unique steering and suspension systems. This experience was exceptional, which allowed the team members to show their engineering skills and participate in the race of space exploration.

\section{CONCLUSION}

DustyTRON team members represented in Fig. 19 showed their skills an interesting real-life challenge. Placing the 29th out of 54 invited universities and getting the "Innovative Design" Award, were a great conclusion for the third-year team performance. The team designed and build a unique robot that represents the mechanical, electrical, and software constraints, by being resourceful and implementing systems engineering principles to solve world-level problems. 


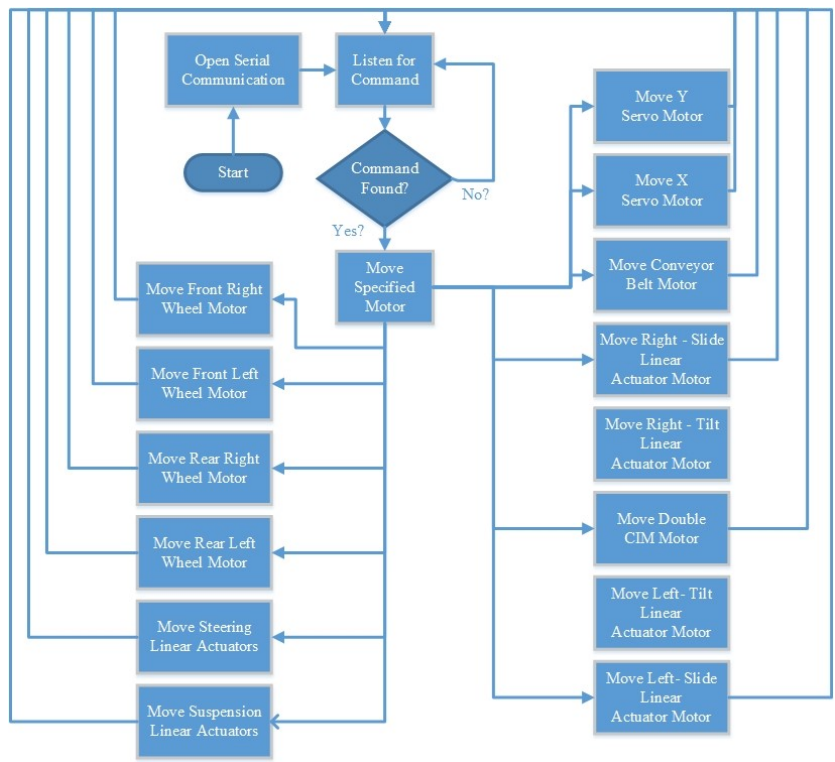

Fig. 17. DustyTRON 3 Robot - Arduino Code Flow Chart.

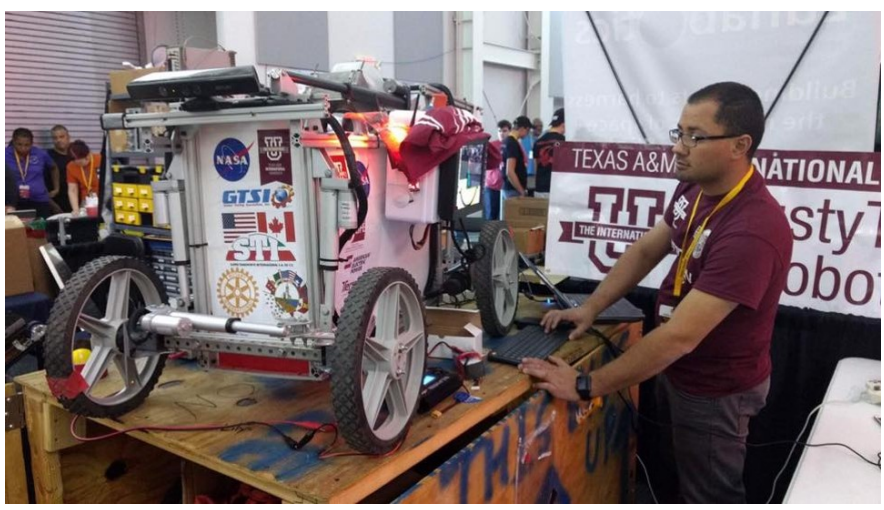

Fig. 18. DustyTRON 3 Robot - Final RMC 2017.

The team strives to improve on team management, time management, and leadership skills. The team will continue seeking new mechanical, circuitry, and software-related concepts to be implemented on future robot designs. Throughout the project, the team has been able to implement engineering skills acquired as Systems Engineering students but also learned how to work as a cohesive team while adding new skills.

For future competitions, the team will practice the continuous improvement principles to learn from their mistakes and develop a better robot:

- Enhance the autonomous operation by utilizing new computer vision algorithms.

- Improve the excavations system to include auger and conveyor system.

- Enhance the adaptive suspension and steering systems to be more compatible with harsher terrain.

The team was inspired to share this experience with local students and the community, hosting STEM days at

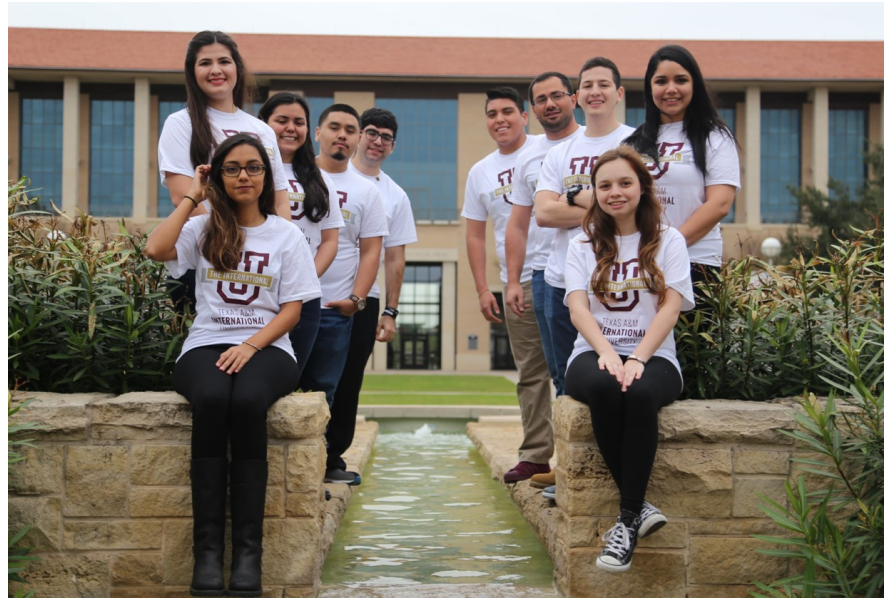

Fig. 19. DustyTRON 3 Team Members.

local schools promoting the interest in the robotics field and NASA's programs and projects. Additionally, the team took advantage of all the venues to support and mentor local FIRST Lego/Tech/Robotics teams and host their local competitions.

\section{ACKNOWLEDGMENT}

Thanks to NASA for providing such a great opportunity to participate in such a competition. This project would not be successful without the support of Texas A\&M International University (TAMIU) and our sponsors from Laredo, TX.

\section{REFERENCES}

[1] Voosen, Paul. "Mars rover steps up hunt for molecular signs of life." (2017). Science 03 Feb 2017, Vol. 355, Issue 6324, pp. 444-445, DOI: 10.1126/science.355.6324.444.

[2] Koris, Daniel R., and Jason Isaacs. (2017) "A Formal Approach to Extended State Machines for Multi-Objective Robots Operating in Dynamic Environments." Proceedings of the 2017 Midstates Conference on Undergraduate Research in Computer Science and Mathematics.

[3] Tashtoush, T., Velazquez, A., Aranguren, A., Cavazos, C., Reyes, D., Hernandez, E., Bueno, E., Otero, E., Zamudio, G., Casarez, H., Rullan, J., Rodriguez, J., Villarreal, J. C., Gutierrez, M., Rodriguez, P., Torres, R., Martinez, R., and Partida, S., "Developing a Mining Robot for Mars Exploitation: NASA Robotic Mining Competition (RMC)", International Journal of Advanced Computer Science and Applications(IJACSA), 11(12), 2020. http://dx.doi.org/10.14569/IJACSA.2020.0111205.

[4] Tashtoush, T., Hernandez, R., Yanez, R., Gonzalez, J., Moreno, H., and Escobar, V. (2020). "Reverse-Twister Swarm Search Algorithm Design: NASA Swarmathon Competition", International Journal of Research Studies in Computer Science and Engineering (IJRSCSE), 7(1), pp.13-20.

[5] Hernandez, R., Yanez, R., Gonzalez, J., Moreno, H., Escobar, V., and Tashtoush. T., (2016) "Design of a Swarm Search Algorithm: DustySWARM Reverse-Twister Code for NASA Swarmathon." Texas A\&M International University, School of Engineering.

[6] Tashtoush, T., Gutierrez, O., Herrera, E., Medina, J., Peña, A., Varela, E., and Hernandez, R. (2020). "Design of a Swarm Search Algorithm: DustySWARM Spiral Epicycloidal Wave (SEW) Code for NASA Swarmathon", International Journal of Research Studies in Computer Science and Engineering (IJRSCSE), 7(1), pp.28-36.

[7] Gutierrez, O., Herrera, E., Medina, J., Peña, A., Varela, E., Hernandez, R., and Tashtoush. T. (2017) "Design of a Swarm Search Algorithm: DustySWARM Spiral Epicycloidal Wave (SEW) Code for NASA Swarmathon". Texas A\&M International University, School of Engineering.

[8] Tashtoush, T., Ruiz, C., Estevis, T., Herrera, E., Bernal, R., Martinez, R., and Reyna, L. (2020). "Square Spiral Search (SSS) Algorithm for Cooperative Robots: Mars Exploration", International Journal of Research Studies in Computer Science and Engineering (IJRSCSE), 7(1), pp.21-27. 
[9] Ruiz, C., Estevis, T., Herrera, E., Bernal, R., Martinez, R., Reyna, L. and Tashtoush, T., (2018) "Design of a Swarm Search Algorithm: Square Spiral Search (SSS) Algorithm for NASA Swarmathon". Texas A\&M International University, School of Engineering.

[10] Tashtoush, T., Ahmed, J., Arce, V., Dominguez, H., Estrada, K., Montes, W., Paredez, A., Salce, P., Serna, A., and Zarazua, M., "Developing a Radiating L-shaped Search Algorithm for NASA Swarm Robots", International Journal of Advanced Computer Science and Applications (IJACSA), Volume (11), Issue (8), August 2020. http://dx.doi.org/10.14569/IJACSA.2020.0110802.

[11] Ahmed, J., Arce, V., Dominguez, H., Estrada, K., Montes, W., Paredez, A., Salce, P., Serna, A., Zarazua, M., and Tashtoush, T. "Developing a Radiating L-shaped Search Algorithm for NASA Swarm Robots", Texas A\&M International University, School of Engineering.

[12] Secor, P. (2016). "NASA Swarmathon".

[13] Braccio, M. (2019). Design of a Robot for the 2019 NASA Robotic Mining Competition. In Proceedings of the Wisconsin Space Conference (Vol. 1, No. 1).

[14] Neubert, J. J. (2016). Using NASA's Robotic Mining Competition to Give Students a Quality Systems Engineering Experience. In ASEE's 123rd Annual Conference \& Exposition (pp. 4-11).

[15] Guerra,L., Murphy, G., and May. L., (2013). Applying Engineering to the Lunabotics Mining Competition Capstone Design Challenge. Proceeding of the ASEE Annual Conference and Exposition, June 2013.

[16] Stecklein, J. (2017, July). NASA's Robotic Mining Competition Provides Undergraduates Full Life Cycle Systems Engineering Experience. In INCOSE International Symposium (Vol. 27, No. 1, pp. 1456-1473).

[17] Berrios, D. C., Galazka, J., Grigorev, K., Gebre, S., \& Costes, S. V. (2020). NASA Genelab: Interfaces for the Exploration of Space OMICS data. Nucleic Acids Research, 2020 Oct 20. https://doi.org/10.1093/nar/gkaa887.

[18] Balint, T. S., Kolawa, E. A., Cutts, J. A., \& Peterson, C. E. (2008). Extreme environment technologies for NASA's robotic planetary exploration. Acta Astronautica, 63(1-4), 285- 298. https://doiorg.tamiu.idm.oclc.org/10.1016/j.actaastro.2007.12.009.

[19] Bogue, R. (2012). Mars curiosity: sensors on the red planet. Sensor Review, 32(3), 187-193. https://doiorg.tamiu.idm.oclc.org/10.1108/02602281211233151.

[20] Cohen, B. A., Chavers, D. G., \& Ballard, B. W. (2012). NASA'S Robotic Lunar Lander Development Project. Acta Astronautica, 79, 221-240. https://doi.org.tamiu.idm.oclc.org/10.1016/j.actaastro.2012.03.025.

[21] Cole, T. J., Bassler, J., Cooper, S., Stephens, V., Ponnusamy, D., Briere, M.,\& Betenbaugh, T. (2012). The challenges of designing a lightweight spacecraft structure for landing on the lunar surface. Acta Astronautica, 71, 83-91. https://doiorg.tamiu.idm.oclc.org/10.1016/j.actaastro.2011.08.003.

[22] Chaput, A., 2016, 'System Engineering Education for All Engineers - A Capstone Design Approach'. ASEE 123rd Annual Conference \& Exposition, New Orleans, June 26-29, 2016.

[23] Mahmood, M. 2016, 'Oakton Community College 2016 NASA Robotic Mining Competition Systems Engineering Paper', paper presented to the 2016 NASA Robotic Mining Competition, Kennedy Space Center, Florida, 16-20 May.

[24] The University of Alabama in collaboration with Shelton State Community College, 2016, 'Journey to Mars; 2016 Systems Engineering Paper', paper presented to the 2016 NASA Robotic Mining Competition, Kennedy Space Center, Florida, 16-20 May.

[25] Charlotte 49er Miner Robotics, The University of North Carolina at Charlotte, 2016, '2016 Systems Engineering Paper', paper presented to the 2016 NASA Robotic Mining Competition, Kennedy Space Center,
Florida, 16-20 May.

[26] Illinois Robotics in Space (IRIS), the University of Illinois at UrbanaChampaign, 2016, 'Design and Development of the IRIS-6 Robotic Mining System', paper presented to the 2016 NASA Robotic Mining Competition, Kennedy Space Center, Florida, 16-20 May.

[27] John Brown University Eaglenaut Robotics, John Brown University, 2015, 'Robotic Regolith Excavation System', paper presented to the 2015 NASA Robotic Mining Competition, Kennedy Space Center, Florida, 1822 May.

[28] Chicago EDT Robotics, the University of Illinois at Chicago, 'Systems Engineering Report 2016, the University of Illinois at Chicago, AMES-3 Surus', paper presented to the 2016 NASA Robotic Mining Competition, Kennedy Space Center, Florida, 16-20 May.

[29] Iowa State University Cyclone Space Mining, '2015-2016 Systems Engineering Paper', paper presented to the 2016 NASA Robotic Mining Competition, Kennedy Space Center, Florida, 16-20 May.

[30] Dieter, G. E., \& Schmidt, L. C. (2013). Engineering design. Boston: McGraw-Hill Higher Education.

[31] "Rules and Rubrics", Nasa.gov, 2017. [Online]. Available: http://www. nasa.gov/offices/education/centers/kennedy/technology/nasarmc/RulesR ubricsResources

[32] Kapurch, S. J. (Ed.). (2010). NASA systems engineering handbook.National Aeronautics and Space Administration. Diane Publishing.

[33] Bellestri, S., Boil, T., Carswell III, M., et al. (2013). Alabama Lunabotic 2013 Systems Engineering Paper (Undergraduate Thesis) Retrieved from NASA.

[34] Alfaro, D., Aranguren, A., Duarte, T., De La Cruz H., Perez, G., Torres, A. Delgado, J., Melero, D., Vazquez, J. A., Charlton, B., Jose Guajardo, J., Flores, G., Garza, E., \& Tashtoush, T. (2015). Systems Engineering Paper (Undergraduate Thesis) Retrieved from Texas A\&M International University School of Engineering.

[35] Ay, N., Bertschinger, N., Der, R., Güttler, F., \& Olbrich, E. (2008). Predictive information and explorative behavior of autonomous robots. The European Physical Journal B, 63(3), 329-339.

[36] Shotts Jr, W. E. (2012) "The Linux command line: a complete introduction" No Starch Press.

[37] Carson, E. M., Rivadeneira, J., Woodward, N. K., \& Peterson, P. W. (2016). "NASA Robotic Mining Competition 2015-2016".

[38] Mueller, R. P. (2012) "Lunabotics Mining Competition: Inspiration through Accomplishment" Thirteenth ASCE Aerospace Division Conference on Engineering, Science, Construction, and Operations in Challenging Environments, and the 5th NASA/ASCE Workshop On Granular Materials in Space Exploration.

[39] Williams, W. B., \& Schaus, E. J. (2015). Design and Implementation of a Rocker-Bogie Suspension for a Mining Robot. In ASEE Southeast Section Conference.

[40] Liu, Y., Jeremy B., Zachary C., Jennifer B., John A.s, Madelyn D., David S., Cindy L. B., John B., and Christopher A.. "Mechanical design, prototyping, and validation of a Martian robot mining system." SAE International Journal of Passenger Cars-Mechanical Systems 10, no. 201701-1305 (2017): 177-182.

[41] Mueller, R., \& Van Susante, P. (2011, September). A review of lunar regolith excavation robotic device prototypes. In AIAA SPACE 2011 Conference \& Exposition (p. 7234).

[42] A Brief Introduction to TurboVNC. (2016, February 20). http://www.turbovnc.org/About/Introduction.

[43] Mojtahedzadeh, R. (2011). Robot obstacle avoidance using the Kinect. Master of Science Thesis Stockholm, Sweden. http://urn.kb.se/resolve?urn=urn:nbn:se:kth:diva-130746.

[44] Arduino MEGA 2560 \& Genuino MEGA 2560. (2017) https://www.arduino.cc/en/Main/arduinoBoardMega2560. 